\title{
Effect of fresh water supply on agriculture in the southwest of the Netherlands
}

\author{
P. J. M. van Boheemen ${ }^{1}$, P. J. Kusse ${ }^{2}$, C. Maas ${ }^{3}$ and J. W. Wesseling ${ }^{4}$ \\ 1 Institute for Land and Water Management Research (ICW), Wageningen, \\ Netherlands \\ 2 Government Service for Land and Water Use, Utrecht, Netherlands \\ 3 Provincial Water Authority of Zeeland, Middelburg, Netherlands \\ 4 Delft Hydraulics Laboratory, Emmeloord, Netherlands
}

Received 31 March 1983; accepted 12 April 1983

Key-words: water management, water requirement, sprinkling, flushing, farm economics

\section{Summary}

In order to get data for the decision upon the management of some lakes in the southwestern part of the Netherlands, investigations are underway to obtain an insight in the effect of water supply on agriculture. The results obtained in the first phase for a 15000 ha region are discussed.

In the study the increase in crop yields due to overhead sprinkling was determined. Next the total water requirement for both sprinkling and flushing of the water courses for quality control was derived. Finally the economic effects of water supply for agriculture were evaluated.

The average increase in crop yields due to sprinkling irrigation is $15-20 \%$ for potatoes, fruit trees and grass, $10-15 \%$ for sugar-beets, leguminous plants, cereals, onions, celeriac and bulbs and less than $5 \%$ for chicory, winter carrots and leek. The maximum water requirement for sprinkling and flushing amounts to about 3.4 $\mathrm{mm} \mathrm{d}^{-1}$. For adaption of the surface water system an investment of $f$ (Dutch guilders) 20-25 million is necessary. The yearly net profit for the farmers lies between $f 3$ and 4 million.

\section{Introduction}

In 1953 the Netherlands were badly hit by floods. Large parts of the southwest were inundated by the North Sea. To avoid the possibility of such a calamity in future, the government decided to enforce the dikes along the North Sea and to close some sea arms. Due to this so-called Delta Plan a number of lakes has been formed. In the original plan these lakes were meant as reservoirs for fresh water to be used for agriculture. 
In the meantime plans have been changed. The Easterscheldt (see Fig. 1) will obtain an open connection and its water will remain salt for fishery and environmental purposes. The Lake Grevelingen contains at the moment salt water but can be made fresh in future. If so, this lake could play an important role in the water supply for agriculture in the adjacent areas. The former island Schouwen Duiveland on the south side of the lake (Fig. 1) then can be provided with fresh water by direct intake from the lake. If the lake water will remain salt, water supply is only possible by transporting water to the island from other bassins.

As part of an investigation into the future management of the lakes in the southwest, the effect of water supply on the agriculture of the island Schouwen Duiveland was analysed. The present paper reports about this study.

\section{Increase in crop yields by fresh water supply}

\section{Calculation model}

The crops on Schouwen Duiveland often suffer from insufficient water supply. The reduction in yield corresponds with the magnitude of the occurring water deficiency. In the study the water deficiency has been determined as the difference between actual and potential evapotranspiration.

De Laat (1980) developed a model to compute the actual water use of crops. In this model the transient flow of water in the soil profile is simulated by a succession of steady states with a duration of 10 days. For this purpose the soil profile is divided into a root zone, an unsaturated and a saturated subsoil. The root zone is defined as the layer from which the crop withdraws the water. Because of the predominant influence of the roots, the presence of hydraulic gradients and consequent fluxes in the root zone is ignored. Due to soil water suctions caused by water uptake in the root zone, water is transported vertically upward in the subsoil. These fluxes are computed from hydraulic gradients and conductivities at the prevailing water contents.

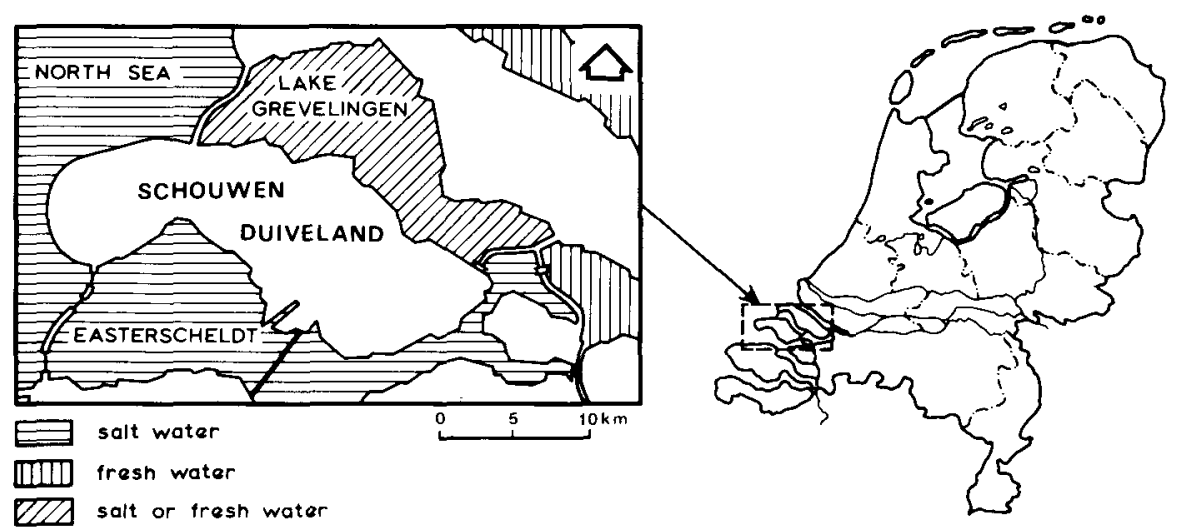

Fig. 1. Situation of Schouwen Duiveland in the southwestern part of the Netherlands. 
The water uptake by the crop is set equal to the potential evapotranspiration as long as the soil water pressure is above a certain critical value, and equal to zero if the soil water pressure is below $-1600 \mathrm{kPa}(\mathrm{pF} 4.2)$. For soil water pressures in the intermediate range the actual evapotranspiration is derived by interpolation using a semilogarithmic relation.

The saturated subsoil represents the upper part of the groundwater storage and can be fed and depleted by transport of water from, respectively to deep aquifers and the surface water system (subsurface irrigation).

To apply the model data on the initial soil water content, the physical properties of the different soil layers and the fluxes through the upper and lower boundaries of the profile are required.

\section{Input data}

Data on free water evaporation and precipitation in the study area were available for the summers (April-September) of the years 1933-1980. The potential evapotranspiration $E_{\mathrm{p}}$ for the different crop types was derived from the free water evaporation $E_{\mathrm{o}}$ (Penman) by using the formula $E_{\mathrm{p}}=f \cdot E_{\mathrm{o}}$. Values for the crop factor $f$ were taken from literature.

In order to account for variations in crop and soil type the study area was divided into squares of 25 ha. For each element a representative crop type was determined from existing maps. The crop types distinguished were: potatoes, sugar-beets, cereals, leguminous plants, onions, chicory, winter carrots, celeriac, leek, bulbs, fruit trees and grass. The total area of these crops amounts to about 15000 ha. For each crop type a value for the critical soil water pressure was assumed together with a value for the thickness of the root zone.

Similarly a representative soil type was determined for each element. To reduce the possible number of combinations of soil and crop types the soil types distinguished on the soil map were grouped in 11 units for which the relations between soil water pressure and soil water content, and those between soil water pressure and hydraulic conductivity could be derived on the basis of structural and textural properties.

The flow between the saturated subsoil and the surface water system was simulated by a drainage function. All agricultural soils on the island were drained by pipes, situated at $0.9 \mathrm{~m}$ below surface during the reclamation after the floods of 1953. The discharge from the drains has been supposed to be proportional to the average depth of the groundwater table using a zero discharge at a groundwater depth of $0.9 \mathrm{~m}$ and a discharge of $8 \mathrm{~mm} \mathrm{~d}^{-1}$ at a groundwater depth of $0.3 \mathrm{~m}$. Seepage of ditch water into the drains does not occur because all the drains are situated above ditch water level. Direct drainage to and seepage from the ditches have been ignored because of the great distances between the ditches and the low transmissivity of the soils.

The geohydrological profile in the study area is characterized by a covering layer of clay and peat with a thickness of about $9 \mathrm{~m}$ and below that a more permeable sandy aquifer. For the description of the flow between the shallow groundwater in the covering layer and the deep groundwater results from a geohydrological study 
could be used. This means that for each element a value for the positive (upward) or negative (downward) seepage was available.

Computations were made for summer periods, starting with an initial soil water content equal to the average situation in the field at 1 April, i.e. a water-table equal to the average groundwater depth in spring and an equilibrium moisture profile in the unsaturated zone.

\section{Results of model computation}

For each 25 ha element and each summer of the period 1933-1980 the actual water use was calculated. Next the water deficiencies were determined assuming that the water requirement of a crop equals potential evapotranspiration. The deficiencies obtained for the elements with the same crop type and the different summers were averaged. Expressed in percentages of the average potential evapotranspiration in the summers 1933-1980 the following values of the average water deficiency were computed: potatoes $18 \%$, sugar-beets $10 \%$, cereals $10 \%$, leguminous plants $15 \%$, onions $12 \%$, chicory $4 \%$, winter carrots $3 \%$, celeriac $13 \%$, leek $2 \%$, bulbs $10 \%$, fruit trees $20 \%$ and grass $16 \%$.

To transform the water deficiency into a yield reduction it was assumed that the relation between crop yield and actual water use can be approximated by a linear function and that sprinkling with an amount equal to the water deficiency will reduce the yield reduction to zero. This means that the values given above also indicate the increases in crop yield by additional water supply.

\section{Water requirement for sprinkling and flushing}

\section{Areal distribution of seepage}

Because part of the area is below sea level and in most of the area ditch water levels below mean sea level are maintained, there is a continuous saline seepage from the sea. This causes the water in the ditches to be brackish. This will remain so when fresh water is supplied, unless flushing is applied with an extra amount of fresh water above the requirement to cover the water deficiencies of the crops.

In order to compute the amount of water for flushing the areal distribution of the seepage was simulated for stationary flow with the computer programme GROMULA of the Delft Hydraulics Laboratory (Broks \& Dijkstra, 1979). GROMULA is a Galerkin finite element programme for flow in a multi-layered system of aquifers.

Fig. 2 gives the element grid system used in the study. On the basis of geohydrological information available for the area, the geohydrological profile was schematized into two separate aquifers divided by a semi-pervious layer and a semi-pervious covering layer on top. The boundary conditions for the model were a constant water level (mean sea level) along the boundary of the area and a constant polder level in the upper semi-pervious layer.

Field data on piezometric levels in both aquifers were used for model calibration. Moreover, the model results could be verified with water balance computations over several years, available for the greater part of the island. With the model the 


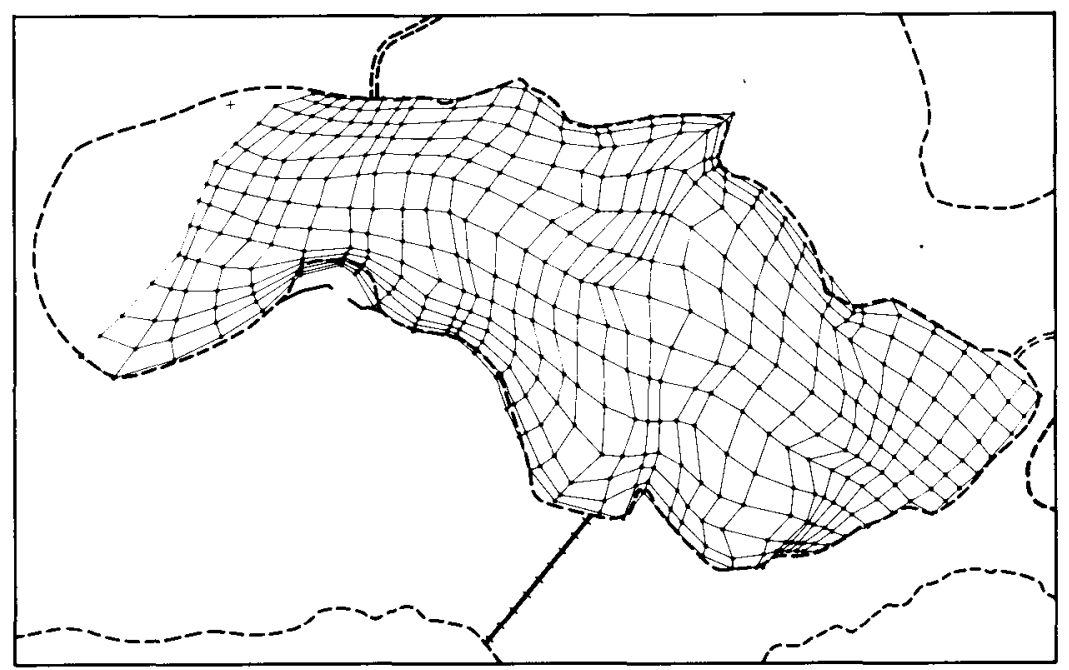

Fig. 2. Element grid system for the model to compute the areal distribution of the seepage.

areal distribution of the stationary seepage was computed for the future situation with ditch water levels that have to be realized when water is supplied to the area. Fig. 3 shows the average daily seepage from the aquifer into the top layer.

\section{Chloride load of the ditches}

Although the seepage of salt water from the sea into the area is a more or less steady process, the flow of saline groundwater to the ditches shows marked fluctuations in time. This phenomenon is caused by the storage of salt in the semi-pervious top layer during summer due to a shortage of rainfall. There appears to exist a close relation between the transport of salt towards the ditches and the difference between precipitation and actual water use by the crops. Because in the study data on the salt load of the ditches had to be known to derive the flushing requirement, a special computer programme LINMOD was developed and applied to each element of the GROMULA model. Essentially LINMOD describes the discharge of water from the soil surface to the ditches through the soil profile. Because the problem does not require a comprehensive knowledge of the processes acting in the plant-soil system, both the crop and the unsaturated zone are modeled very schematically.

Fig. 4 shows the set-up of LINMOD. From field observations it was concluded that after each rainshower some rapid downward flow takes place, presumably through cracks. In the scheme this effect is indicated as 'short circuiting'. The inflow from the deeper aquifers into the semipervious top layer may take place either towards the drains or towards the unsaturated zone. In both cases the relation between inflow and outflow of the saturated zone can be expressed in the form

$$
F_{\text {out }}=\alpha \int_{o}^{t} F_{\text {in }}(t-\tau) \mathrm{e}^{-\alpha \tau} d \tau
$$




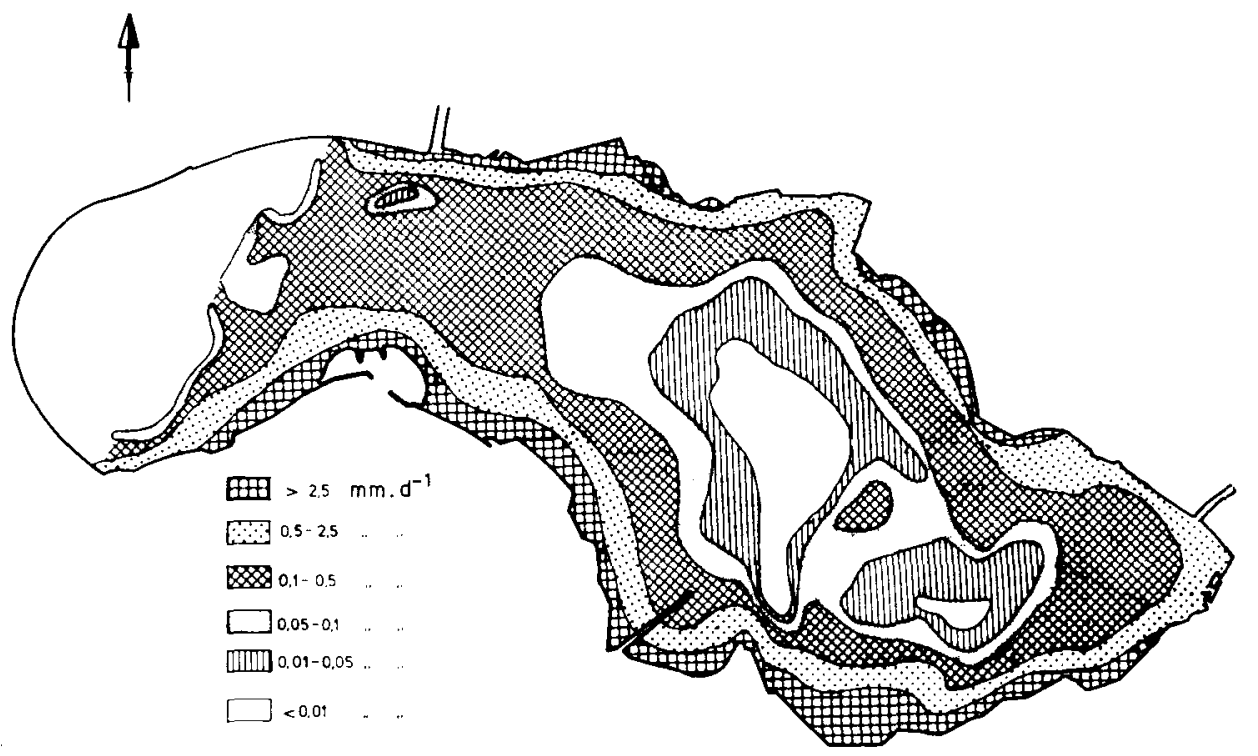

Fig. 3. Computed areal distribution of the average daily seepage at raised ditch water levels.

where $\alpha$ forms the reaction factor which depends on drainage intensity and soil properties. For situations with a phreatic level above the drains the value of $\alpha$ valid for the pipe drainage was taken, while for situations with a phreatic level below the pipe drains the $\alpha$ value for the ditch system was used.

To convert water discharges into salt loads, the discharges of the drains were multiplied by the chloride content of the drainwater and the direct discharges from the subsoil to the ditches by the chloride content of the water in the upper aquifer.

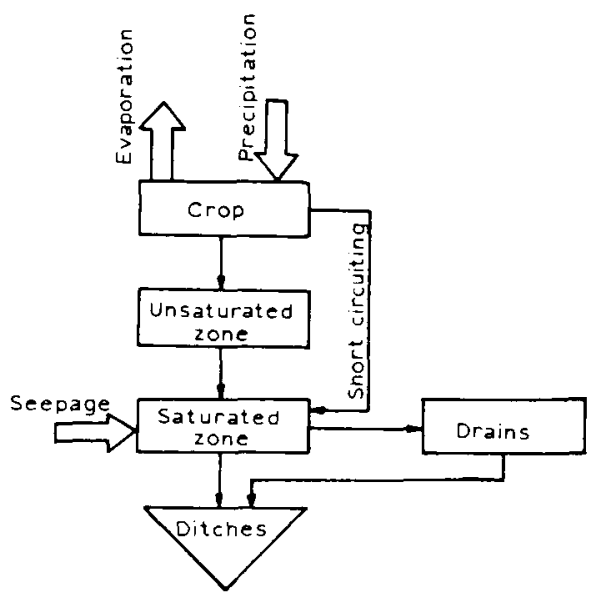

Fig. 4. Set-up of the model LINMOD. 


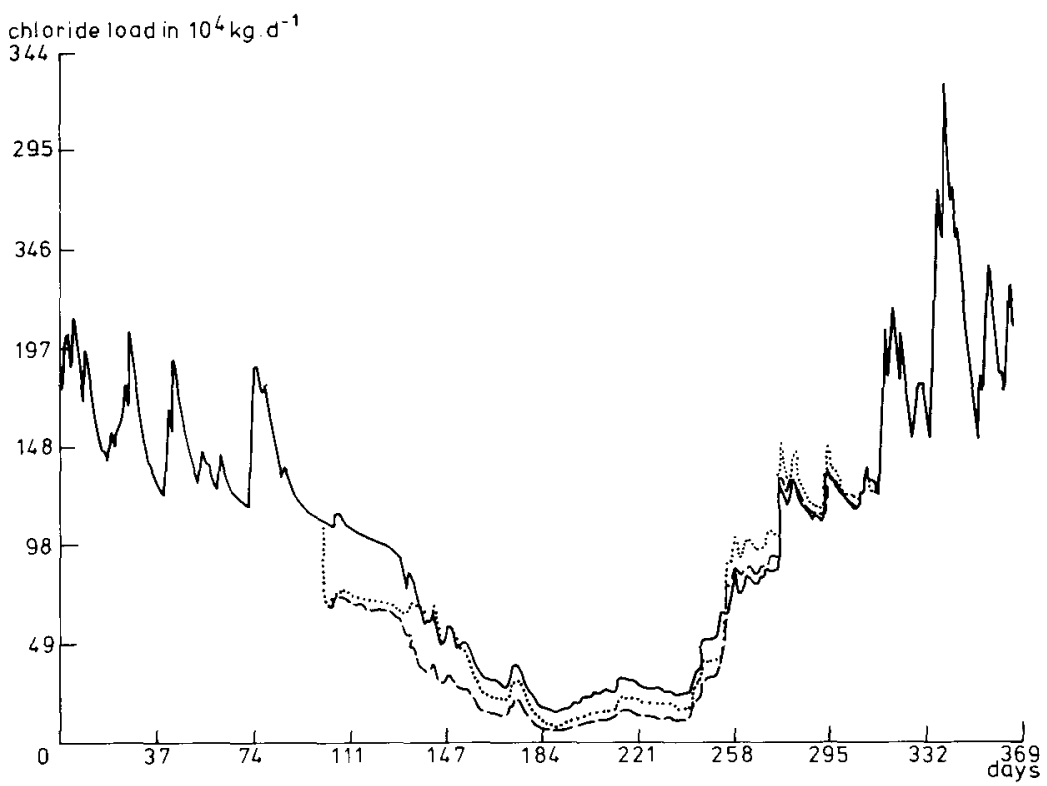

Fig. 5. Computed temporal fluctuation of the chloride load of the surface water in 1976. chloride load at present ditch water levels;

- - . - chloride load at raised ditch water levels;

. . . . . . chloride load at raised ditch water levels and apply of sprinkling.

For both parameters data were available from earlier investigations.

LINMOD was calibrated for a sequence of years with data available for the area. Figs. 5 and 6 present examples of the results. Fig. 5 gives the time-dependent chloride load of the ditches for the whole area. When the polder levels are raised during summer the discharge of salt decreases. This effect is partly canceled when sprinkling is applied. Fig. 6 gives the areal distribution of the salt load of the ditches at the start of the growing season of 1976 when ditch water levels would have been raised and sprinkling would have been applied.

\section{Maximum water requirement}

The highest additional water requirement for flushing and sprinkling together appeared to occur at the beginning of a dry growing season like the summer of 1976. Therefore the areal distribution of the salt load on the ditches given in Fig. 6 was taken as starting point for the computations of the maximum water requirement. An additional condition for computing the water requirement was, that the chloride content in the ditch water must be kept below $1 \mathrm{~kg} \mathrm{~m}^{-3}$. Further it was assumed that sprinkling takes place with water from side branches of the surface water system and the optimal situation is reached when the quality standard is just met at the confluences of the side branches and the main canals. As water needed for sprinkling also helps to combat salinization of the ditch water, no distinction has been made between water for sprinkling and water for flushing. 


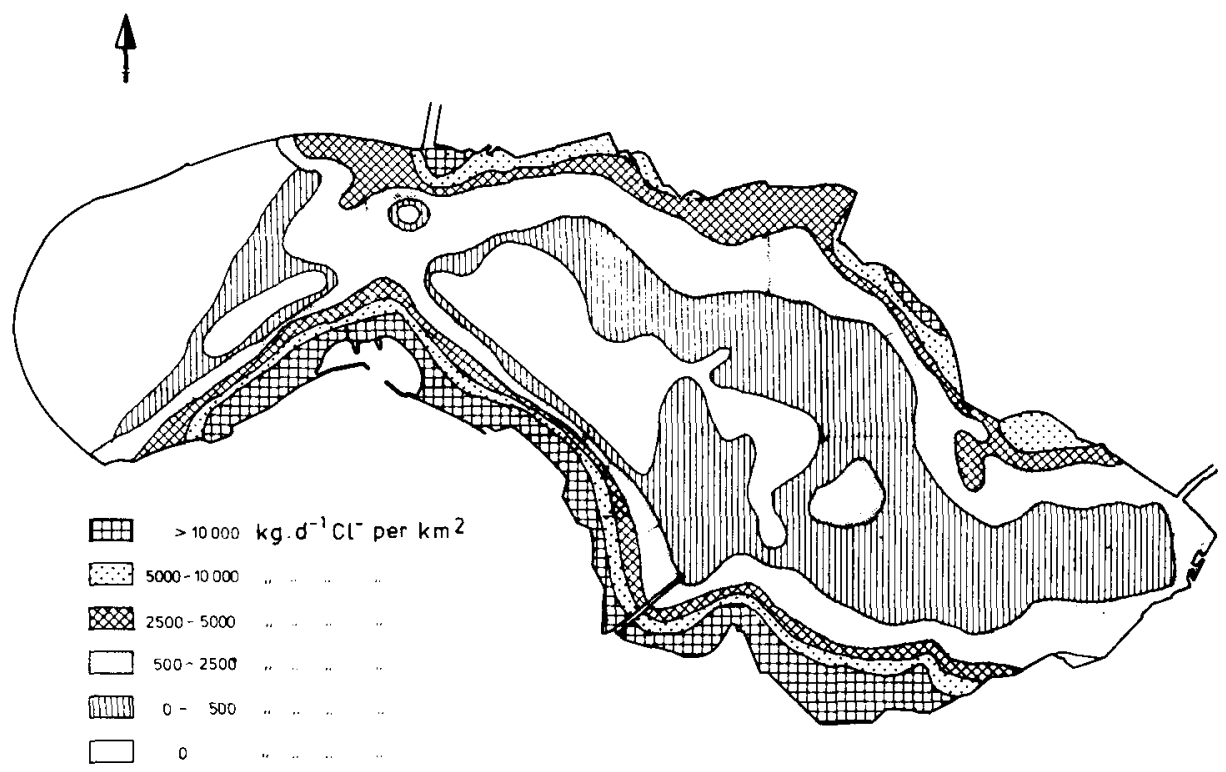

Fig. 6. Computed spatial distribution of the chloride load on the surface water in April/May 1976 if the ditch water levels would have been raised and sprinkling would have been applied.

Concerning the requirement for sprinkling the assumption was made that in the critical period sprinkling with an intensity of $3 \mathrm{~mm} \mathrm{~d}^{-1}$ occurs on one third of the cultivated area.

Calculations were carried out for two different supply systems, one with a diffuse intake from Lake Grevelingen and the other with water from another basin transported by a pipeline to a location at the east side of the island. For both cases the total amount of water needed for flushing and sprinkling together is $3.4 \mathrm{~mm} \mathrm{~d}^{-1}$ of which $1 \mathrm{~mm} \mathrm{~d}^{-1}$ is used for sprinkling. If some very saline areas are excluded from flushing, this amount may be lowered to $2.5 \mathrm{~mm} \mathrm{~d}^{-1}$. Compared with other coastal areas of the Netherlands where fresh water is supplied regularly, the computed amounts are extremely high. The reason for this must be sought in the severe salinization of the island Schouwen Duiveland.

\section{Economic effects}

Realization of the fresh water supply requires inlet works and enlarging of the present drainage canals and ditches. Based on the needed transport capacity as determined for the critical period, the total investment for construction of these works in case of a diffuse intake from Lake Grevelingen amounts to $f 22.3$ million, which may be lowered to $f 18.4$ million when some very saline areas are excluded from water supply. The variant with a pipeline for fresh water to the east side of the island requires $f 24.3$ million, which can be reduced to $f 20.0$ million. In these 
amounts the investment for the construction of the pipeline is not included.

The net profit of fresh water supply for the farmers was computed by means of an optimalization model developed by the Research Station for Arable Farming and Field Production of Vegetables (PAGV). For a small part $(10 \%)$ of the area with fruit culture, bulb culture and dairy farming this model could not be applied. For these crops a very simple method was used in which the increase in yield was directly converted into benefits.

For the net profit attainable four scenarios have been considered, namely:

PS- $\quad$ : present situation without sprinkling;

PS+ : present situation with sprinkling;

OPT- : optimum situation without sprinkling;

OPT + : optimum situation with sprinkling;

For the two OPT scenarios farm plans were obtained from the optimalization mod$\mathrm{el}$, with the net profit as a criterion for the optimalization and farm size, labour costs, machinery costs, crop rotation requirements, yield reductions and crop prices as input data. In order to quantify the effects of changes in the farm plan (cropping pattern) due to fresh water supply the model was applied on farm level.

As mentioned the annual fixed and variable costs of sprinkling formed input data for the model. The capacity of the sprinkling installation was set at 30 or $60 \mathrm{~m}^{3}$ hour ${ }^{-1}$ and the annual fixed costs at $20 \%$ of the replacement value. This $20 \%$ consists of interest, depreciation, maintenance and insurance. The variable costs, mainly costs of energy, have been based on the gross water gift, the capacity of the installation, the price of gas oil and the area with sprinkling. The accounted total sprinkling costs vary from $f$ 467-691 ha- year ${ }^{-1}$. Comparing these figures with the actual and potential crop yields revealed that sprinkling is only profitable for potatoes, onions, celeriac, sugar-beets and chicory.

Table 1 shows the acreages used for agriculture and horticulture under the different scenarios. For the scenarios PS- and PS + the figures were obtained from inventories on farming. They pertain to the present crop acreages in the study area. The figures for the scenarios OPT- and OPT + were computed with the optimalization model and represent the cropped acreages when farmers would grow other crops because of the higher amount of money gained from these crops. Realization of

Table 1. Acreage with agriculture and horticulture under the different scenarios as a percentage of the total area with both cultures.

\begin{tabular}{llll}
\hline & \multicolumn{2}{l}{ Scenario } & \\
\cline { 2 - 4 } & PS- and PS+ & OPT- & OPT + \\
Agriculture & 89 & 72 & 68 \\
Horticulture & 11 & 28 & 32 \\
Total & 100 & 100 & 100 \\
\hline
\end{tabular}


Table 2. Yearly increase in income of the farmers by fresh water supply in million Dutch guilders.

\begin{tabular}{lll}
\hline & Minimum effect & Maximum effect \\
Agriculture and horticulture & 2.50 & 3.10 \\
Fruit culture & 0.60 & 0.60 \\
Bulb culture & 0.15 & 0.15 \\
Dairy farming & 0.01 & 0.10 \\
Total & 3.26 & 3.95 \\
\hline
\end{tabular}

these simulations depends on the market possibilities of the products. The table shows that a shift in crops forms a possibility to raise the net incomes. After optimalization of the farm plans, there will be a smaller shift in crops upon introduction of sprinkling.

Table 2 presents the total yearly increase in income of the farmers by water supply. In this table the profits for the fruit culture, bulb culture and dairy farming are mentioned too. The so-called minimum effect was derived by comparing the incomes of the farmers computed for the scenarios PS- and PS+; the so-called maximum effect by comparing the incomes for the scenarios OPT- and OPT + .

The minimum effect for agri- and horticulture of $f 2.5$ million year ${ }^{-1}$ corresponds with an increased net yield of $f 4100$ year $^{-1}$ for a 20 -ha farm and $f 9800$ year $^{-1}$ for a 40-ha farm.

The sprinkling costs amount to $f 3.6$ million year ${ }^{-1}$, thus the gross minimum effect of sprinkling amounts to $f 6.9$ million year ${ }^{-1}$ and the gross maximum effect to $f 7.6$ million year ${ }^{-1}$.

The real economic effect of the water supply will be higher than indicated by the above figures, because a multiplier effect has not been taken into account.

\section{References}

Broks, A. P. M. \& D. Dijkstra, 1979. GROMULA, numeriek model voor de berekening van potentialen en debieten bij grondwaterstromingen welke voldoen aan de wet van Darcy. Delft Hydraulics Laboratory, Delft, Netherlands.

Laat, P. J. M. de, 1980. Model for unsaturated flow above a shallow water table applied to a regional sub-surface flow problem. Agric. Res. Rep. 895. Pudoc, Wageningen, Netherlands, 126 pp. 